\title{
Measurements of isolated prompt photons in pp collisions with the ATLAS detector
}

\author{
Francesco POLCl ${ }^{* \dagger}$ \\ LPSC/IN2P3/CNRS \\ E-mail: francesco.polciecern.ch
}

\begin{abstract}
Measurements of the cross sections for the inclusive productions of isolated prompt photons and di-photons in proton-proton collisions at a centre-of-mass energy $\sqrt{s}=7 \mathrm{TeV}$ at the Large Hadron Collider (LHC) with the ATLAS detector are presented. Photon candidates are identified by combining information from the calorimeters and from the inner tracker. Residual background in the selected sample is estimated from data based on the observed distribution of the transverse isolation energy in a narrow cone around the photon candidate. The results are compared to predictions from next-to-leading order perturbative QCD calculations.
\end{abstract}

The 2011 Europhysics Conference on High Energy Physics, EPS-HEP 2011,

July 21-27, 2011

Grenoble, Rhône-Alpes, France

\footnotetext{
*Speaker.

On behalf of the ATLAS collaboration
} 


\section{Introduction}

The measurements of the cross sections of isolated prompt photons and di-photons are of interest as probes of the perturbative QCD predictions. In addition they provide an estimate of the background for important searches at LHC like the ones for the Higgs boson, the Randall-Sundrum gravitons and the signature of Universal Extra-Dimensions with two photons in the final state.

Prompt photons come from the hard scattering subprocess of $p p$ collisions, mainly $q g \rightarrow q \gamma$ at the LHC, so they are sensitive to the gluon density function. An additional contribution is due to photons originating from the fragmentation of a high transverse momentum $p_{T}$ parton.

The di-photon final state occurs mainly through the $q \bar{q} \rightarrow \gamma \gamma$ and the box diagram $g g \rightarrow \gamma \gamma$, the latter giving a contribution comparable to the former given the large gluon flux at the LHC. The contribution from $q \gamma$, production with isolated photon radiation from the quark is also considered.

ATLAS measured these cross sections using $p p$ collisions at a centre-of-mass energy $\sqrt{s}=7$ $\mathrm{TeV}[1,2,3]$. The inclusive isolated prompt photon production cross section as function of the photon transverse energy $E_{T}$ measurement used first an integrated luminosity of $(0.88 \pm 0.1) \mathrm{pb}^{-1}$ covering the range $15<E_{T}<100 \mathrm{GeV}$ and $|\eta|<1.81^{1}$ [1], and then (34.6 1.2$) \mathrm{pb}^{-1}$ for the range $45<E_{T}<400 \mathrm{GeV}$ and $|\eta|<2.37$ [2]. The isolated di-photon production cross section has been measured with $37 \mathrm{pb}^{-1}$ as function of the di-photon invariant mass $m_{\gamma \gamma}$, the transverse momentum $p_{T \gamma \gamma}$ and the azimuthal angular separation $\Delta \phi_{\gamma \gamma}$, for photons with $E_{T}>16 \mathrm{GeV}$ and $|\eta|<2.37$ [3].

\section{Photon reconstruction and identification in ATLAS}

The ATLAS detector at the LHC is described in detail in $[4,5]$. A photon deposits its energy in the Liquid Argon (LAr) electromagnetic calorimeter, covering the region $0<|\eta|<1.37$ and $1.52<|\eta|<2.37$. For the $\sim 30 \%$ of photons converting in $e^{+} e^{-}$pairs before reaching the calorimeter, the reconstructed tracks are matched to the calorimeter deposit for a complete kinematical reconstruction of the photon. The reconstruction efficiency is $\sim 85 \%(\sim 75 \%)$ for $0<|\eta|<1.37$ $(1.52<|\eta|<2.37)$, the main losses being due to dead readout modules currently recovered.

The main challenge for the photon identification is to reject neutral hadrons carrying most of the energy of a jet. For this purpose ATLAS defines a number of variables exploiting the longitudinal and lateral segmentation of the calorimeter to provide a description of the shower profile, expected to be much narrower and well contained in the LAr for a $\gamma$ than for a hadron. In particular the fine $\eta$ granularity of the first compartment of the LAr allows to resolve the two maxima typical of the energy deposit of a $\pi^{0}$. The selection criterion on these variables used in the analysis is called tight [1] and is optimized separately for converted and unconverted photons. Its efficiency varies depending on $\eta$ and increasing with $E_{T}$.

Another key variable is the calorimeter transverse energy isolation $E_{T}^{i s o}$, which is the sum of the energies of the cells in a cone of radius $\Delta R=\sqrt{(\Delta \eta)^{2}+(\Delta \phi)^{2}}=0.4$ around the direction of

\footnotetext{
${ }^{1}$ The ATLAS reference system is a Cartesian right-handed coordinate system, with the nominal collision point at the origin. The anticlockwise beam direction defines the positive $z$-axis, while the positive $x$-axis is defined as pointing from the collision point to the centre of the LHC ring and the positive $y$-axis points upwards. The azimuthal angle $\phi$ is measured around the beam axis, and the polar angle $\theta$ is measured with respect to the $z$-axis. Pseudorapidity is defined as $\eta=-\ln \tan (\theta / 2)$
} 
the photon, minus the energy deposited by the photon candidate itself as well as the soft jet activity of the underlying event [6]. It is weakly correlated to the tight identification criterion.

\section{Event selection}

To ensure that the selected event is coming from a collision, all tracks in the event are analyzed to search for at least one primary vertex with three or more tracks.

In the single photon analysis, a fully efficient high level trigger with energy threshold $10 \mathrm{GeV}$ for [1] and $40 \mathrm{GeV}$ for [2] is used. A total of 110 thousand photons for [1] and 174 thousand for [2] pass the tight requirement and have $E_{T}^{i s o}<3 \mathrm{GeV}$.

In the di-photon analysis, a fully efficient di-photon trigger with $15 \mathrm{GeV}$ threshold per photon is applied. The two most energetic tight photon candidates with $E_{T}>16 \mathrm{GeV}$ and separated by a $\Delta R>0.4$ are retained, the first being referred to as the leading and the other as the subleading photon. The selected sample contains 2022 events with $E_{T}^{i s o}<3 \mathrm{GeV}$ for each photon.

\section{Background subtraction methods}

In the single photon analysis, two methods have been used to subtract the background : the two-dimensional sideband method and the template method [1,2]. In the sideband method, the events populate a two-dimensional plane formed by the variables $E_{T}^{i s o}$ and tight criterion. Using some reference cuts on these variables, four regions A, B, C, D can be identified, the signal being mostly confined in region A. In the hypothesis of low correlation for the background and low signal leakage from region A, one can easily extract the number of photons from the relation $N_{A}^{s i g}=N_{A}-N_{B} \frac{N_{C}}{N_{D}}$. Corrections for signal leakage and residual correlations are properly taken into account. In the template method, the isolation distributions of tight photons is fitted. The background shape is determined from a background enriched control sample of photon candidates not passing the identification criteria, while the signal shape is taken from electrons from $W$ and $Z$ decays, correcting for the difference with photons using simulations.

In the di-photon cross section measurement, the sidebands method is iterated on the leading and subleading photon and the template method fits the two-dimensional distribution of the leading versus subleading $E_{T}^{i s o}$. The additional approach of the matrix is also used [3]. In this technique each event is classified in four categories according to whether each of the tight photon candidates passes or not the isolation criteria. The categories are related to four weights describing how likely the event belongs to each category through a matrix of the efficiency for a photon or a jet to pass or not the isolation cut, measured from data. Giving to each event the corresponding weight, the signal can be extracted. Correlations in the $E_{T}^{i s o}$ of the two photon candidates are taken into account.

The electron contamination is evaluated from data control samples and subtracted too. The background subtraction procedures are iterated in bins of $\eta$ and $p_{T}$, and show a good agreement $[1,2,3]$.

\section{Results}

Knowing the integrated luminosity, the signal yields extracted with the background subtraction and the efficiencies from signal simulation, the cross section can be measured. Correction 
factors taking into account the migration between neighboring bins due to the energy resolution are included as measured with unfolding techniques from simulations. Results are shown on figures 1 and 2, including in the error bands the systematic uncertainties. Correlated uncertainties have been treated consistently. For the reconstruction efficiency the main uncertainties come from the isolation efficiency cut (3-4\%) and the limited knowledge of the detector material (1-2.5\%). For the identification efficiency the systematic uncertainty ranges from $1.5 \%$ to $3 \%$ depending on $\eta$, with contributions from the detector material knowledge, the background contamination, conversion classification, fragmentation contribution and the Monte Carlo generators. The yields determination has a total systematic uncertainty of $10 \%$ decreasing with $E_{T}$ due to inputs from the simulations, the choice of the background control region and the photon energy scale (2-8\%)

For the single photon analysis the result is compared to JETPHOX predictions [7], using CTEQ 6.6 PDFs, setting the scale to $E_{T}^{\gamma}$ and applying an isolation cut at parton level of $4 \mathrm{GeV}$ in a cone of radius 0.4 . The scale uncertainty $(10 \%)$ is the leading theoretical systematic uncertainty together with the PDFs uncertainty (5\%) and the choice of the parton level isolation criterion (2\%). Results show, in general, a good agreement. Nevertheless for $E_{T}<35 \mathrm{GeV}$, where the contribution from fragmentation is higher, the theory [7] seems to overestimate data. For the di-photon analysis the comparison is done with DIPHOX [8] and ResBos [9]. Some deviations are observed for low $\Delta \Phi_{\gamma \gamma}$ values, related to the region $m_{\gamma \gamma}<2 E_{T}^{c u t}$ (being $E_{T}^{c u t}=16 \mathrm{GeV}$ ). For these values the $g g$ box diagram dominates. More accurate predictions (NNLO) would provide clarifications on these residual discrepancies.

\section{Conclusions}

Using $p p$ collisions at a centre-of-mass energy $\sqrt{s}=7 \mathrm{TeV}$, ATLAS measured the inclusive isolated prompt photon production cross section as function of the photon transverse energy $E_{T}$ : first in the range $15<E_{T}<100 \mathrm{GeV}$ and $|\eta|<1.81$ with an integrated luminosity of $(0.88 \pm 0.1) \mathrm{pb}^{-1}$ and then in the range $45<E_{T}<400 \mathrm{GeV}$ and $|\eta|<2.37$ using (34.6 \pm 1.2$) \mathrm{pb}^{-1}$. It also measured the isolated di-photon production cross section as function of the di-photon invariant mass $m_{\gamma \gamma}$, the transverse momentum $p_{T \gamma \gamma}$ and the azimuthal angular separation $\Delta \phi_{\gamma \gamma}$, for photons with $E_{T}$ $>16 \mathrm{GeV}$ and $|\eta|<2.37$ with $37 \mathrm{pb}^{-1}$. Results are in agreement within errors with the current predictions available. More accurate theoretical predictions will help to understand the hints of possible deviations in the $d \sigma / d E_{T}$ distribution for $E_{T}<35 \mathrm{GeV}$ and for low values of $\Delta \Phi_{\gamma \gamma}$. 

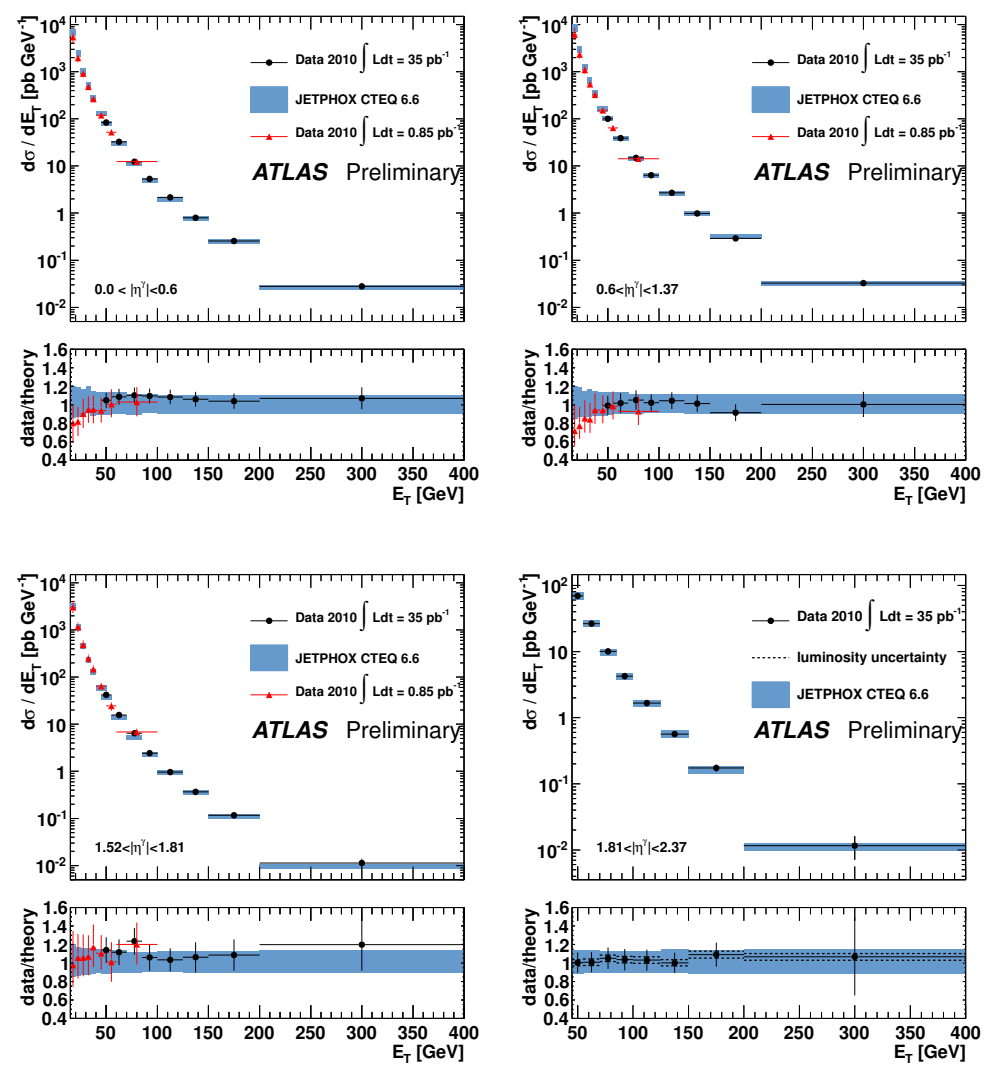

Figure 1: Measured versus expected (JETPHOX CTEQ6.6) differential cross-section $d \sigma / d E_{T}$ of inclusive isolated prompt-photon production in different pseudorapidity ranges.
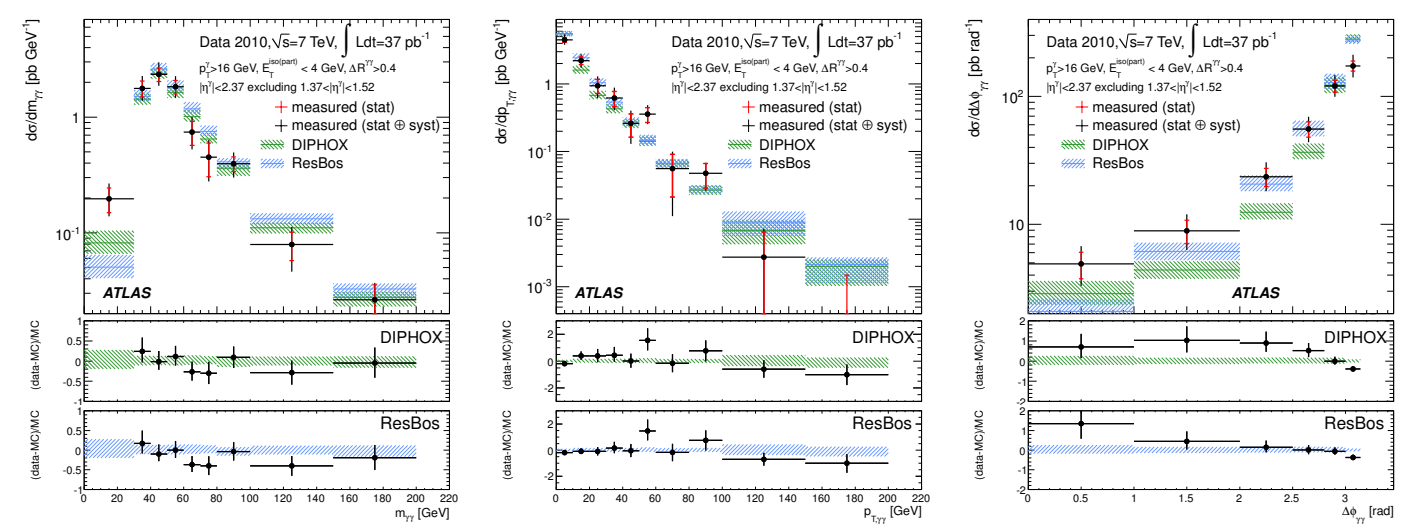

Figure 2: Measured versus expected (DIPHOX, ResBos) differential cross-sections $d \sigma / d m_{\gamma \gamma}, d \sigma / d p_{T, \gamma \gamma}$ and $d \sigma / d \Delta \Phi_{\gamma \gamma}$ of di-photon production. 


\section{References}

[1] G. Aad et al. Phys. Rev. D83 (2011) 052005. [arXiv:1012.4389 [hep-ex]].

[2] G. Aad et al. [arXiv:1108.0253 [hep-ex]], submitted to Phys. Lett. B.

[3] G. Aad et al. [arXiv:1107.0581 [hep-ex]], accepted by Phys. Rev. D.

[4] G. Aad et al. JINST 3 (2008) S08003.

[5] G. Aad et al. [arXiv:0901.0512 [hep-ex]].

[6] M. Cacciari, G. P. Salam, S. Sapeta, JHEP 1004 (2010) 065. [arXiv:0912.4926 [hep-ph]].

[7] M. Fontannaz, J. P. Guillet, G. Heinrich, Eur. Phys. J. C21 (2001) 303-312. [hep-ph/0105121].

[8] T. Binoth, J. P. Guillet, E. Pilon, M. Werlen, Eur. Phys. J. C16 (2000) 311-330. [hep-ph/9911340].

[9] C. Balazs, E. L. Berger, P. M. Nadolsky, C. -P. Yuan, Phys. Rev. D76 (2007) 013009. [arXiv:0704.0001 [hep-ph]]. 